\title{
MORTALIDAD MATERNO PERINATAL EN HUANCAYO, 2001-2004
}

\section{MATERNAL MORTALITY PERINATAL IN HUANCAYO, 2001-2004}

\author{
Gustabo Acevedo Gonzalez' Liliana Munive Lázaro' Norma Jáuregui Villar ${ }^{2}$
}

\section{RESUMEN}

La mortalidad materna perinatal presenta indicadores negativos de la salud reproductiva de la Región y el País. La mortalidad materna indica el estado de salud de un grupo poblacional, sus condiciones económicas, sociales, culturales y la calidad de su atención obstétrica. La investigación tuvo como objetivo: determinar los factores de riesgo que influyeron en el aumento de la mortalidad materna perinatal en los hospitales de la ciudad de Huancayo durante los años 2001 al 2004. El tipo de investigación ha sido la básica explicativa, con diseño histórico lógico, porque se recolectó datos del pasado para describirlo, explicarlo e interpretarlo. Con los resultados obtenidos se concluye que, los factores para la muerte de una mujer por motivo de su función reproductiva son crónicos y trágicos. Esta situación repercute en los estratos socioeconómicos y culturales más bajos, sin acceso a los servicios de salud, grado de instrucción primaria, estado civil soltero y conviviente, con alta paridad y en edad fértil, del ámbito urbano y rural, con control prenatal reducido y la indiferencia de las entidades públicas. La Dirección Regional de Salud Junín, cuenta en Huancayo con deficiente disponibilidad de recursos humanos profesionales y establecimientos de salud.

Palabras clave: Mortalidad materna perinatal, salud reproductiva, edad fértil.

\section{SUMMARY}

The perinatal maternal mortality presents negative indicators of the reproductive health of the Region and the Country. The maternal mortality indicates the state of health of a populational group, its economic, social, cultural conditions and the quality of its obstetric attention. The investigation had as objective: to determine the factors of risk that influenced in the increase of the perinatal maternal mortality in the hospitals of the city of Huancayo during the years 2001 at the 2004. The investigation type has been the basic one explanatory, with logical historical design, because it was gathered data of the past to describe it, to explain to it and to interpret it. With the obtained results you concludes that, the factors for the death of a woman for reasons of their reproductive function are chronic and tragic. This situation rebounds in the lowest socioeconomic and cultural strata, without access to the services of health, grade of primary instruction, single civil state and conviviente, with high parity and in fertile age, of the urban and rural environment, with reduced prenatal control and the indifference of the public entities. The Regional Address of Health Junín, has in Huancayo faulty readiness of professional human resources and establishments of health.

Key words: Perinatal maternal mortality, reproductive health, fertile age. 


\section{INTRODUCCIÓN}

La investigación científica basada en evidencias es uno de los grandes pilares y fuentes del desarrollo de la ciencia, la tecnología y de la sociedad actual y del futuro, (1). La investigación se realizó con el objetivo de determinar los factores que influyeron en el aumento de la mortalidad materna perinatal en pacientes atendidas en los hospitales de la ciudad de Huancayo durante los años: 2001 al 2004. Esta mortalidad es un problema complejo multidimensional.

Cada una constituye un problema de investigación, es decir, es susceptible de investigarse desde el punto de vista biológico, médico, obstétrico, etc. La mortalidad materna en el Perú es de $185 \times 100000$ nacidos vivos/año, una de las más altas de América Latina. La probabilidad de morir por causas maternas es dos veces mayor para las mujeres del área rural que para las mujeres del área urbana. La muerte materna está condicionada a factores como: barreras económicas, geográficas, socioculturales y barreras de conducta de los prestadores de salud. Sumándose a todo ello reducida cobertura de profesionales Obstetras y Gineco-obstetras.

\section{MATERIAL Y MÉTODOS}

El tipo de estudio fue básico explicativo porque se buscó explicaciones o hallazgos de causalidad, de relaciones esenciales y necesarias entre dos variables. Para llegar a conocer los fenómenos y hechos del trabajo. Como método específico se utilizó el método histórico ubicado en el tiempo, para establecer su evolución así como sus antecedentes o factores y repercusiones para el incremento de la mortalidad materna peri natal.

El diseño de la investigación fue histórico y lógico, que permitió la reunión de datos del pasado no sólo para describirlos, sino para explicarlos e interpretarlos tratando de establecer su evolución, sus antecedentes o factores y sus repercusiones. Se tuvo un enfoque dialéctico aplicado a la historia. Aquí se tomó la autenticidad de las fuentes y la validez interna de los contenidos, ya que de ello dependió la objetividad, la verdad y la generalidad de los resultados.

El estudio se realizó en los hospitales, centros y puestos de salud, Instituto de Medicina Legal, la Dirección Regional de Salud, Fiscalía provincial, Colegios Profesionales, Iglesias, las Universidades y el Ministerio de Educación de la provincia de Huancayo. La unidad de análisis estuvo conformada por toda la documentación y versiones directas de familiares de madres fallecidas en los establecimientos de salud de la provincia de Huancayo durante los años 2001 al 2004.

La técnica de la entrevista se aplicó a los familiares, para obtener información de la condición económica, social, cultural, edad, paridad, grado de instrucción y causa directa de muerte de las madres fallecidas. Asimismo se utilizó el análisis documental de las historias clínicas para contrastar y obtener mejor información al respecto. Para la recolección y procesamiento de datos se elaboraron los instrumentos: la guía de entrevista y la ficha de análisis del contenido de las historias clínicas; se validaron, aplicaron y se analizaron con el uso de técnicas estadísticas descriptivas lo que nos permitió describir, caracterizar y resumir los datos obtenidos

\section{RESULTADOS}

En la Tabla 1, se observa la cifra de 31 muertes maternas en los últimos cuatro años en Huancayo. Estas muertes maternas se deben a causas prevenibles y que pueden ser evitadas con técnicas sencillas y de bajo costo en los primeros niveles de atención.

abla 1. Incidencias de mortalidad materna perinatal en Huancayo, años 2001 al 2004.

\begin{tabular}{|c|c|c|c|c|c|c|c|c|c|c|}
\hline \multirow{2}{*}{$\begin{array}{l}\mathrm{N}^{0} \text { de } \\
\text { casos }\end{array}$} & \multicolumn{8}{|c|}{ Años } & \multicolumn{2}{|c|}{ Total } \\
\hline & 2001 & $\%$ & 2002 & $\%$ & 2003 & $\%$ & 2004 & $\%$ & $N^{\circ}$ & $\%$ \\
\hline $\begin{array}{l}\text { Muerte } \\
\text { Materna } \\
\text { Perinatal }\end{array}$ & 6 & 19 & 10 & 32 & 7 & 23 & 8 & 26 & 31 & 100 \\
\hline Total & 6 & 19 & ข0 & 32 & 7 & 23 & 8 & 26 & 31 & 100 \\
\hline
\end{tabular}

Fuente; Elaboración propia

Tabla 2. Incidencia de muertes maternas perinatales, segün edat en Huancayo, años 2001 al 2004.

\begin{tabular}{|c|c|c|c|c|c|c|}
\hline \multicolumn{7}{|c|}{ Edades (aiios) } \\
\hline Año & 15 a 24 & 25 a 31 & 35 a 44 & 45 a más & $\mathbf{N}^{o}$ & $\%$ \\
\hline 2001 & 3 & 2 & 1 & 0 & 5 & 19 \\
\hline 2002 & 3 & 5 & 2 & 0 & 10 & 32 \\
\hline 2000 & 2 & 3 & 2 & 0 & 7 & 23 \\
\hline 2004 & 2 & 5 & 1 & 0 & 8 & 26 \\
\hline Total & 10 & 5 & 5 & 7 & $\equiv 1$ & $\mathrm{im}$ \\
\hline
\end{tabular}

Fuente: Elaboraz on propia.

En los últimos cuatro años hubieron 15 muertes maternas (48\%) y tenían edades de 25 a 34 años, seguidas de un $36 \%$ entre 15 a 24 años. La mortalidad materna está presente en todas las edades de la fertilidad materna.

Observamos que en Huancayo tiene 139 instituciones relacionadas con la salud, de los cuales el 19 \% realizan actividades de promoción, 
Tabla 3. Distribución de establecimientos de salud, MINSA, Huoncayo; años: 2001 al 2004.

\begin{tabular}{lcc}
\hline Catagaría & Frecuencia & $\%$ \\
\hline Hospitales & 2 & 1 \\
Centros de Salud & 25 & 18 \\
Puestos de Salud & 112 & 81 \\
Total & 139 & 100 \\
\hline
\end{tabular}

Fuerte. Elaboración pıspia.

prevención resolución y rehabilitación de la salud materna. La distribución y el número de establecimientos del Ministerio de Salud no satisfacen las necesidades de la demanda, los cuales se convierten en factores de riesgo en influyen en el incremento de la mortalidad materna.

Tabla A. Recurses humanos probsionales dol MaNSA, que atienden ol emharazo, parto y puerperio, Huaricayo; arius 2001 al 200.1.

\begin{tabular}{lcc} 
Recursus Iltrmanus & Nimern & 5 \\
\hline Mésicos obstetras & 17 & 12 \\
Oustreticesiobstetras & 82 & 88 \\
Total & 73 & 160 \\
\hline
\end{tabular}

Fuerta Llaboracion ororis.

El número de profesionales especialistas en la atención del embarazo, parto y puerperio es escaso, los mismos no satisfacen la demanda de la mujer en su salud reproductiva, existiendo la necesidad de mayor formación y distribución equitativa de dichos profesionales en la región.

Tabla 5. Nùnero de acciones sociales de las instituciones públicas, para disıninuir la mortalidad materna perinatal en Huancayo: aौtos: 2nO1 al 2004.

\begin{tabular}{|c|c|c|}
\hline Instituciones públicas & Frecuencia & * \\
\hline DIS $\lambda_{\text {, Jumin }}$ & 25 & $\Rightarrow$ \\
\hline Ministerio de Ealucaciōin & 5 & 2 \\
\hline Iglesia & 2 & : \\
\hline Colegios profesionales & 5 & \{ \\
\hline Deferrsoria del pueblo & 2 & 5 \\
\hline |u|a| & 12 & 100 \\
\hline
\end{tabular}

Eume: Flist uroriót ampa

En la Tabla 5, se observa que la única institución pública que promociona la salud y previene la mortalidad materna es el Ministerio de Salud (59 $\%)$ mientras las demás instituciones públicas participan en acciones mínimas el cual repercute en la práctica social con altas tasas de mortalidad materna peri natal.

\section{DISCUSIÓN}

La mayoría de los estudios de mortalidad materna perinatal, señalan como causas de muerte materna directas a las hemorragias, abortos, infecciones, hipertensión inducida por la gestación que representan un $85 \%$ y las indirectas el $15 \%$.

La mortalidad materna indica el estado de salud de un grupo poblacional, sus condiciones económicosociales, culturales y la calidad de su atención obstétrica. Esta afirmación es corroborada con la investigación, que ha permitido determinar las causas de mortalidad materna en los hospitales de Huancayo, existiendo 31 muertes maternas entre los años 2001-2004 y que pueden haber sido evitados con técnicas sencillas y de bajo costo en los primeros niveles de atención.

El $48 \%$ de las madres que fallecieron tenían edades de 25 a 34 años. De esto podemos inferir hoy día, la muerte de una mujer por motivo de su función reproductiva es tan anacrónica y trágica.

En lo que respecta el estado civil de las madres fallecidas podemos inferir que el $45 \%$ eran convivientes y el $29 \%$ solteras, esto es consecuencia del liberalismo sexual con alto riesgo de enfermar y morir durante el embarazo, parto y puerperio.

En el grado de instrucción de las madres fallecidas se observa que el $58 \%$ tenían primaria y el $33 \%$ secundaria, de esto podemos inferir que a menor grado de instrucción mayor riesgo de morir. Estos resultados se corroboran con las investigaciones realizadas en los hospitales Cayetano Heredia, Loayza, San Bartolomé y en el hospital "El Carmen" de Huancayo.

En cuanto a la causa final de muertes maternas en Huancayo, el 48 \% fue el shock hipovolémico, seguido de sepsis e insuficiencia cardiorrespiratorio. Según los resultados se tiene que el $71 \%$ del total de las madres fallecidas se produjeron en la etapa del puerperio.

En la salud materna repercuten múltiples factores biológicos, ambientales, económicos y culturales que es preciso modificar para erradicar el analfabetismo, la discriminación, las desigualdades económicas y sociales, y permitir que la mujer participe en las decisiones que incumben a su salud y su destino.

Referente a las instituciones públicas, que realizan acciones sociales para disminuir la mortalidad materna se observa que el $59 \%$ corresponde al Ministerio de Salud, seguida con un $14 \%$ por los colegios profesionales relacionados con la salud materna y el Ministerio de educación con un 12 \% de acciones.

Los establecimientos de salud-Minsa y los recursos 
humanos profesionales que atienden a la mujer en edad fértil son escasos, no implementados y deficiencias en la disponibilidad de recursos humanos.

No existen diferencias significativas a nivel regional y nacional de los factores que influyen para la muerte materna durante el embarazo, parto y puerperio según edad, condición económico-social, estado civil, paridad, grado de instrucción y causa final de muerte materna, asimismo morir por causas maternas es dos veces mayor para las mujeres del área rural que para las mujeres del área urbana.

De 90 a $95 \%$ de estas muertes pueden evitarse mediante intervenciones sencillas y de bajo costo.

\section{REFERENCIAS BIBLIOGRAFICAS}

1. Escalante M. Violencia, salud y desarrollo. Salud de las adolescentes, Lima: Sociedad Peruana de Adolescencia y Juventud, 2002; 193-201.

2. Farro A, Pacheco J. Mortalidad Materna: Experiencia en el Hospital Nacional Edgardo Rebagliatí Martins. Ginecol Obstet. 2003: 49 (1): 18-31.

3. Instituto Nacional de Estadística e Informática. Encuesta demográfica y de salud familiar 2000, Lima: INEI, Mayo 2001.

4. OPS. Mortalidad Materna en América Latina. Edit. Interamericana, México; 2001.

5. Pacheco J. Ginecología y Obstetricia, Tomo ILima-Perú; 2002.

6. Roeders P. Aprendiendo Juntos: Un Diseño de Aprendizaje activo, Lima; 1997.

7. Shumard E. Obstetricia, Argentina; 1995.

8. Villena Y. Anticoncepción de emergencia. En: Carrasco M, ed. Salud de las adolescentes. Lima: Sociedad Peruana de Adolescencia y Juventud. 2002: 255-68.

9. Yamin A E. Castillos de arena en el camino hacia la modernidad. Lima:Centro de la Mujer Peruana Flora Tristán 2003, pp 338.

E-mail: gustaboacevedo@yahoo.es 